\title{
Epilepsy Management during the COVID-19 Pandemic in Epilepsy Monitoring Unit
}

\section{Maryam Izadi Laybidi}

Epilepsy Nurse, School of Nursing, Isfahan University of Medical Sciences, Isfahan, Iran

Correspondence should be addres sed to Maryam Izadi Laybidi, maryam.izadi91@yahoo.com

Received: September 12, 2020; Accepted: September 25, 2020; Published: October 02, 2020

\section{SHORT NOTE}

Coronavirus disease 2019 (COVID-19) is a rapidly spreading infectious disease caused by the novel human coronavirus, severe acute respiratory syndrome coronavirus 2 (SARS-CoV-2) [1]. The symptoms of COVID-19 infection usuallyappear after an incubation period of about five days. The most common symptoms of COVID-19 illness are fever, cough, and fatigue; other symptoms include headache, hemoptysis, and dyspnea, among others. In the most severe cases, patients may develop pneumonia, acute respiratory distress syndrome, acute cardiac problems, and multi organ failure [2]. Iran reported its first confirmed cases of SARS-CoV2 infection on 19 February, 2020. As of 28 April, 2020 there were 5,806 COVID-19 deaths with more than 91,000 confirmed infections in Iran. Strict quarantine measures have not been implemented in response to this outbreak in Iran; however, the government's actions included cancellation of all public events, closure of schools, and shopping centres, and banning of festival celebrations [3].

Epilepsy, a disease characterized by a spontaneous recurrence of unprovoked seizures, is one of the most common chronic neurological conditions. Its prevalence rate is reported to be $0.7 \%-1.0 \%$ with high incidences in elderly people and children; additionally, some patients have comorbidities [4].

Available information on the pandemic suggests that people with epilepsy (PWE) are neither more likely to be infectedby the coronavirus nor are they more likely to have severe COVID-19 manifestations because they suffer from epilepsy. However, management of COVID19 in PWE may be more complicated than that in other individuals [5].

While, so far no therapy has been approved for the treatment of COVID-19, there are more than a handful of therapies under investigation and being used. Therefore, drug-drug interactions between anti-COVID therapies and antiepileptic drugs (AEDs) could pose significant challenges. In addition, cardiac, hepatic, or renal problems, which may happen in patients with severe COVID-19, may require adjustment to AEDs [5]. Certain combinations are not recommended or require greater attention to prevent the inductions of critical comorbidities. Levetiracetam is of interest, as it would not cause interactions with any drug. Drug interactions should be taken into consideration when introducing or adding AEDs. Moreover, some medications for Citation: Mary am Izadi Laybidi, Epilepsy Management during the COVID-19 Pandemic in Epilepsy Monitoring Unit. J Clin Cases Rep 3(S4): $22-24$.

2582-0435/@ 2020 The Authors. Published by TRIDHA Scholars. 
supportive treatment such as antihistamine would reduce seizure threshold [4].

Epilepsy monitoring using long term video electroencephalography (EEG) is a common practice used by epileptologists whentreating patients with medically intractable epilepsy. This is performed in designated epilepsy monitoring units (EMU) with the assistance of physicians, technicians, and nurses. Epilepsy Nurses are a part of professional care system which has a man role to manage and evaluate of patients with complex uncontrolled Epilepsy. These nurses can manage diagnosing and changing antiepileptic drug of patients $[6,7]$.

\section{The Steps of EEG for nurses include}

Nurse must be marking the electrode places with a red pencil. Marked place must be clean with minimally abrasive skin prep. 30 electrodes are used with Collodion glue (with strong odor and without allergy). The nurse activities are controlled with EEG technician every dayfor doing specific activations (photic stimulation and hyperventilation). A nurse is assisted to patients for use of stationary bike pedals. Video EEG is checked every day with epileptologist [6].

When patients come to the hos pital, they should go to:

- Admitting department Kashani hospital.

- After admission, they will go to the EMU at hospital.

- What will happen when 1 get to EMU.

- Before hospitalization, we take PCR test from patients and their caregivers.

- Hospitalize only three patients with negative PCR test results in this unit Therefore, we reduced the number of ward's staff.

- Additionally, we take health history from patients.

- In this monitoring, patients will stay in separate rooms.

- Nurses and caregivers use personal equipment before dealing with the patient.

- $\quad$ physicians, technicians, nurses, patients and care givers use face mask and follow
- Healthprotocol and Socialdistance.

- Meeting patients is forbidden during COVID-19 pandemic in this unit.

- The presence of residents has decreased.

- Online training and commission meetings are held.

- Nurses use the rest room one by one and according to priority.

- There is a hand sanitizer liquid in each room.

- We educate the patient and caregiver about hand hygiene.

- Ward's staff and patients wash their hands regularly.

- We will prepare them. So we can monitor their epilepsy.

There are 3 steps to prepare them:

A nurse will show their room and the unit. The nurse will then ask them some questions.

1. Ask you about their medical history?

Do a physical exam.

Talk to them about the plan for their cure by epileptolgist (the neurologist who specializes in epilepsy).

A nurse will attach small metal disks (electrodes) to their sculp.

They do this with special glue and cream. These electrodes help us to monitor their brain microphone to record their seizures.

2. What will happen when patients get a seizure?

They will push a bottom if you feel a seizure starts recording what is happening. It also sends a signal to the nurses. Nurses use personal protective equipment dealing with patients during seizures.

3. How long will patients stay?

Your stay may be 6 days; you will stay until we can record enough seizures. A caregiver stays with the patient from the beginning to the end of the stay.

4. What should a patient do during their stay?

They will spend most of time in bed or in a comfortable chair. 
They will need to stay in the epilepsy monitoring unit (EMU) area to record seizure.

This is also for their safety.

Every day we will check their electrodes and add cram if needed.

\section{Things to do for them}

- $\quad$ Check them regularly.

- Respond to their call bell.
- Watch you through a monitor.

- Select normal and abnormal EEG.

- The epileptologist will manage your cure and changes their medicines, if needed.

- Doctor may decide to reduce the amount of medicine or cut it out.

- This will give the team the better chance to record seizure [6].

\section{REFERENCES}

1. Adan GH, Mitchell JW, Marson T (2020) Epilepsy care in the COVID-19 era. Clinical Medicine 20(4): e104-e106.

2. Asadi-Pooya AA, Simani L (2020) Central nervous system manifestations of COVID-19: A systematic review. Journal of the Neurological Sciences 431: 116832.

3. Asadi-Pooya AA, Farazdaghi M, Bazrafshan M (2020) Impacts of the COVID-19 pandemic on Iranian patients with epilepsy. Acta Neurologica Scandinavica.

4. Kuroda N (2020) Epilepsy and COVID-19: Associations and important considerations. Epilepsy \& Behavior 108: 107122.

5. Asadi-Pooya AA, Attar A, Moghadami M, et al. (2020) Management of COVID-19 in people with epilepsy: Drug considerations. Neurological Sciences: 1-7.

6. Izadi Laybidi M (2018) The role of special epilepsy nurse in epilepsy monitoring unit. Epilepsy Journal 4(2).

7. Atkinson M, Hari K, Schaefer K, et al. (2012) Improving safety outcomes in the epilepsy monitoring unit. Seizure 21(2): 124-127. 